\title{
Photo-Induced Reduction of Noble Metal Ions to Metal Nanoparticles on Tubular J-Aggregates
}

\author{
Stefan Kirstein,, ${ }^{1}$ Hans von Berlepsch, ${ }^{2}$ and Christoph Böttcher ${ }^{2}$ \\ ${ }^{1}$ Institut für Physik, Humboldt-Universität zu Berlin, Newtonstrasse 15, 12489 Berlin, Germany \\ ${ }^{2}$ Forschungszentrum für Elektronenmikroskopie, Freie Universität, Fabeckstrasse 36a, 14295 Berlin, Germany
}

Received 6 June 2006; Revised 23 November 2006; Accepted 1 December 2006

Palladium and silver nanoparticles are formed on the surface of tubular J-aggregates of an amphiphilic tetrachlorobenzimidacar-

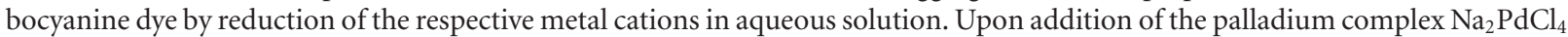
to the aggregate solution, the absorption spectrum shows significant changes which is explained by partial destruction of the aggregates. Cryogenic transmission electron microscopy (cryo-TEM) images show that the tubular J-aggregates are randomly covered by well-separated Pd nanoparticles of approximately 1-3 nm size. Larger particles and higher particle density along the aggregates are obtained when an auxiliary reducing agent is added to the solution. The presence of the metallic particles leads to efficient fluorescence quenching giving clear evidence for super quenching. In similar experiments using $\mathrm{AgNO}_{3}$, silver nanoparticles are grown which are larger in size but less dense distributed along the aggregates. At least in the case of the silver particles, the spontaneous formation of metal nanoparticles is assumed to be initiated by a photo-induced electron transfer process (PET).

Copyright (C) 2006 Stefan Kirstein et al. This is an open access article distributed under the Creative Commons Attribution License, which permits unrestricted use, distribution, and reproduction in any medium, provided the original work is properly cited.

\section{INTRODUCTION}

Since their discovery in the thirties of the last century $[1,2]$, J-aggregates (or Scheibe-aggregates) of cyanine dyes are a fascinating topic of research because of their outstanding optical properties. The spectroscopic peculiarities are the result of exceptionally strong electronic interactions between the transition dipole moments of the dyes which give rise to extended exciton states after photo excitation [3, 4]. The excitonic absorption spectrum depends on the details of the structural arrangement of the dye molecules within the aggregate $[5,6]$. A red-shift of the excitonic absorption with respect to the monomer absorption is considered as the characteristic feature of J-aggregates. In this case, fluorescence emission is observed in resonance to the lowest energy exciton state. The extended exciton states give rise to many peculiar linear and nonlinear optical properties, such as resonant fluorescence [1,2], super radiant emission [7-9], high nonlinear susceptibilities [10], efficient exciton-exciton annihilation [11], and energy migration [12-15]. The latter was already observed by Scheibe et al. [16]. They concluded from quenching experiments on J-aggregates of pseudoisocyanine (PIC) that one quencher molecule per $10^{3}$ to $10^{6}$ dye molecules was sufficient to reduce the fluorescence by $50 \%$ which they explained by migration of excitation energy.
Nowadays, this phenomenon is often called super quenching and a significant subject of research not only in dye aggregated structures $[17,18]$, but also in other materials such as conjugated polymers [19]. However, in most cases, the detailed molecular structure of the aggregated system is either unknown or, as in case of conjugated polymers, amorphous. For a quantitative analysis of the experiments, one has to assume that the quenchers are distributed homogeneously within the aggregates which may not be true for many systems, because the quenchers are immiscible with the ordered phase of the dye aggregates. For more detailed studies of the energy migration effect, a system is desired, where the structure of the aggregates is well defined and where the quenchers can be localized within the aggregates by microscopic techniques.

Recently, it was shown by Dähne et al. that amphiphilic cyanine dyes are able to form very regular and well-defined structures in aqueous solutions already at low concentrations [20-24]. These dyes consist of a 5, 5'-6, $6^{\prime}$-tetrachlorobenzimidacarbocyanine chromophor [20] to which alkyl chains at the $1,1^{\prime}$ position of the nitrogen atoms and different acidoor sulfoalkyl substituents at the $3,3^{\prime}$ position (cf. Scheme 1) have been attached. The length of the alkyl chains was selected in a range, which is short enough (less then dodecyl) to maintain water solubility, but is sufficient (larger than hexyl) 


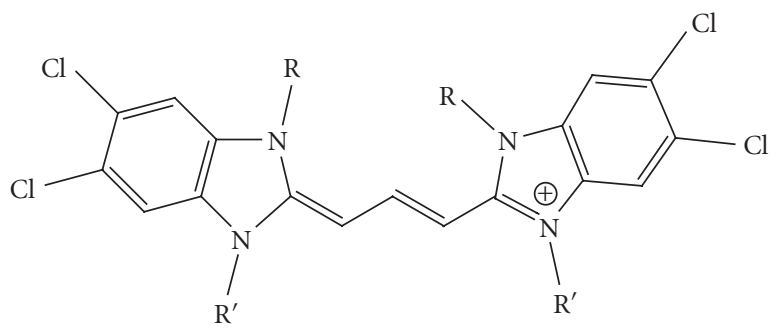

Scheme 1: 5, 5' $-6,6^{\prime}$-tetrachlorobenzimidacarbocyanine, C8S3: $\mathrm{R}=\mathrm{C}_{8} \mathrm{H}_{17}, \mathrm{R}^{\prime}=\mathrm{C}_{3} \mathrm{H}_{6}-\mathrm{SO}_{3} \mathrm{Na}$.

[24] to stabilize the structures by the hydrophobic effect. Many of the dyes are forming tubular structures, which are either dispersed as isolated entities in the aqueous solvent or which are attached to one another to form helically twisted bundles. For the investigations presented here out of various amphiphilic dyes, the dye C8S3 (cf. Scheme 1) was selected because it forms very regular tubular J-aggregates in a concentration range which is on the one hand suitable for spectroscopic investigations, but on the other hand low enough to provide aggregates that are isolated from each other [25].

A very new approach to perform quenching experiments using metal nanoparticles as quenching agents was tried and is reported in this paper. Any attempts to directly adsorb preformed metal nanoparticles such as gold colloids at the aggregates failed. However, it was possible to produce the particles in situ by reduction of noble metal ions (Palladium or Silver) from a respective salt. The reduction of the metal ions may be performed by addition of a special electron donating substance, a so-called reductor. Such a procedure is well known as electroless deposition and of wide technical importance for metal decoration of plastics [26]. In addition, this process is used in basic science and technology [27]. Under certain circumstances the reduction process can also be driven by photo-induced electron transfer processes (PET). Here it will be demonstrated that the reduction of metal cations can be used to decorate the tubular J-aggregates of amphiphilic cyanine dyes with $\mathrm{Pd}$ and $\mathrm{Ag}$ nanoparticles and that these particles serve as fluorescence quenchers. It was found that the aggregates themselves may act as "reductors."

\section{EXPERIMENTAL}

The dye C8S3 was purchased from FEW Chemicals (Wolfen, Germany) as a betain salt and was used as received. The aggregate solutions were prepared as follows: the dye was dissolved in $\mathrm{MeOH}$ at a concentration of several $10^{-3} \mathrm{M}$ and then diluted with pure water (purified by Millipore filter system) for aggregation. These mixtures were then vigorously shaken to become homogeneous and stored without stirring in the dark for 24 hours. The resulting solutions (approximately $4 \times 10^{-4} \mathrm{M}$ dye concentration) are slightly opalescent, but no hint of residual undissolved or precipitated dye material was observed. The content of $\mathrm{MeOH}$ in such solutions is commonly in the order of $15 \mathrm{wt} \%$.
$\mathrm{MeOH}, \mathrm{Na}_{2} \mathrm{PdCl}_{4}$, and $\mathrm{AgNO}_{3}$ were purchased from Aldrich and used as received. The reducing agent was a commercial product (ATOTECH, reductor 406). The absorption spectra were measured with a Lambda 9 spectrophotometer (Perkin Elmer), the fluorescence spectra with a luminescence spectrometer LS 50 B (Perkin Elmer). The samples for cryo-TEM were prepared at room temperature by placing a droplet $(10 \mu \mathrm{L})$ of the solution on a hydrophilized perforated carbon filmed grid ( $60 \mathrm{~s}$ Plasma treatment at $8 \mathrm{~W}$ using a BALTEC MED 020 device). The excess fluid was blotted off to create an ultra-thin layer (typical thickness of $100 \mathrm{~nm}$ ) of the solution spanning the holes of the carbon film. The grids were immediately vitrified in liquid ethane at its freezing point $\left(-184^{\circ} \mathrm{C}\right)$ using a standard plunging device. The vitrified samples were transferred under liquid nitrogen into a Philips CM12 transmission electron microscope using the Gatan cryo-holder and stage (Model 626). Microscopy was carried out at $-175^{\circ} \mathrm{C}$ sample temperature using the microscopes low-dose protocol at a primary magnification of 58300x. The defocus was chosen in all cases to be $0.9 \mu \mathrm{m}$, which corresponds to a first zero of the phase contrast transfer function at $1.8 \mathrm{~nm}$.

\section{RESULTS AND DISCUSSION}

In Figure 1, a cryo-TEM image of aggregates of the sulfonated $5,5^{\prime}-6,6^{\prime}$-tetrachlorobenzimidacarbocyanine C8S3 is presented together with respective absorption and fluorescence spectra. The aggregates are prepared in a $10^{-5} \mathrm{M}$ aqueous solution containing $15 \mathrm{wt} \%$ of $\mathrm{MeOH}$. Under these conditions tubular aggregates are observed with a well-defined diameter of $13 \pm 0.5 \mathrm{~nm}$ and lengths up to several hundreds of nanometers. The weak bending of tubules and the rarely observed assembly into bundles (one is seen in the middle of Figure 1(a)) indicate that they are rather stiff. The tube diameter appears to be highly constant not only along one tube but also between different tubes. Due to the amphiphilic nature of the dye molecules, they are arranged within the wall of tubes as a molecular bilayer. These tubular structures are stable over a few days after preparation, but may undergo structural changes if stored for longer times [28]. The corresponding absorption and fluorescence spectra are shown in Figures 1(b) and 1(c). The absorption is characterized by an excitonic spectrum, that is, split into five distinct peaks (bands I-V) and in total red-shifted compared to the monomer absorption (indicated by the dashed line). The various peaks in the absorption spectrum belong to different excitonic transitions which result from the special molecular packing within the tubular structure. This type of spectrum is typical for the tubular aggregates of differently substituted $5,5^{\prime}-6,6^{\prime}$-tetrachlorobenzimidacarbocyanine dyes, although the peaks may occur at different positions. According to a structure model published recently by Didraga et al. [25], the wall of the tubular aggregates is formed by a double layer of dye molecules and therefore the aggregates may be described by two interleaving tubes of different diameter. Each of the dye layers contributes to the exciton spectrum with two optical transitions. The strongest bands I and II of the absorption 


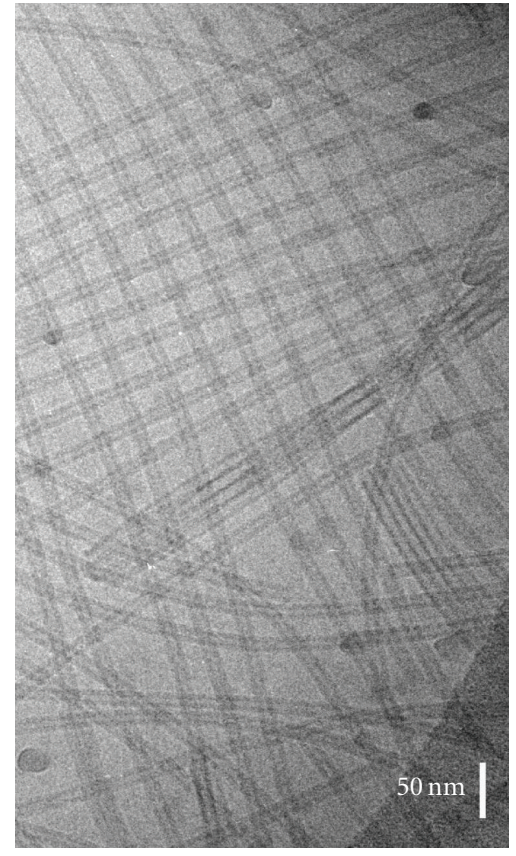

(a)

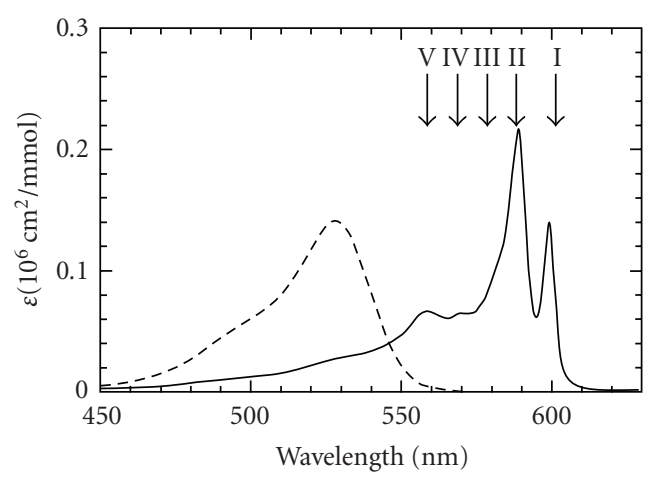

(b)

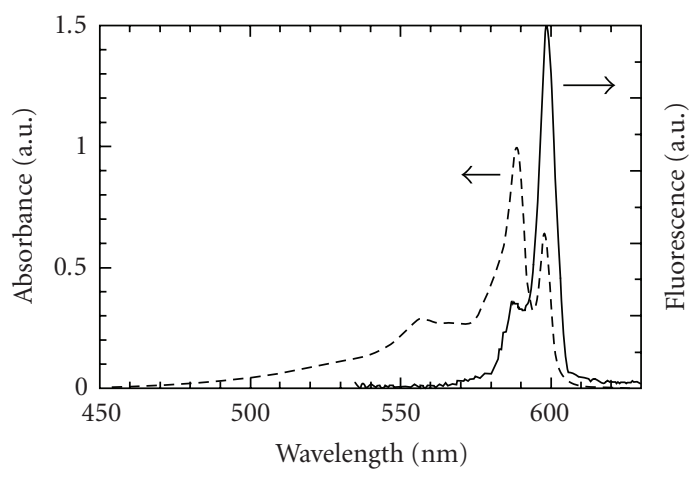

(c)

FIGURE 1: Optical spectra and cryo-TEM images of aggregates of $\mathrm{C} 8 \mathrm{~S} 3$ in water with 16 wt $\%$ of $\mathrm{MeOH}$ added. (a) Cryo-TEM images of a $5.3 \times 10^{-4} \mathrm{~mol} \cdot \mathrm{l}^{-1}$ dye solution. The bar indicates $50 \mathrm{~nm}$. (b) Absorption spectra of a $3.8 \times 10^{-4} \mathrm{~mol} \cdot \mathrm{l}^{-1}$ dye solution. The dashed line represents the absorption of monomers. (c) Fluorescence emission (solid line) and excitation spectra (dashed line) of a $10^{-6} \mathrm{~mol} \cdot \mathrm{l}^{-1}$ dye solution.

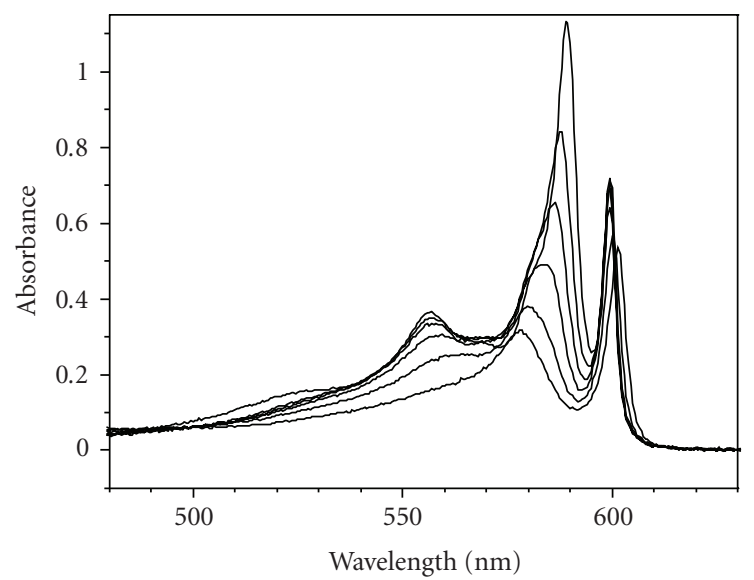

Figure 2: Absorption spectra of a $5 \times 10^{-6} \mathrm{M}$ solution of C8S3 upon titration with $\mathrm{Na}_{2} \mathrm{PdCl}_{4}$ under presence of the reducing agent 406 (ATTOTEC). End concentration of $\mathrm{Na}_{2} \mathrm{PdCl}_{4}: 6 \times 10^{-6} \mathrm{~mol} \cdot \mathrm{l}^{-1}$, titration step: $1 \times 10^{-6} \mathrm{~mol} \cdot \mathrm{l}^{-1}$.

spectrum belong to the inner and outer layers of the wall, respectively, and are polarized in parallel to the long axis of the aggregate, while the weaker transitions on the blue side of the spectrum are polarized perpendicular.

The fluorescence of the tubular aggregates is emitted primarily from the transition at the lowest energy, that is, the band at approximately $600 \mathrm{~nm}$. A small additional contribution is observed from higher states, which indicates that an excitation into higher exciton states is not completely transferred into the lowest energy state. This incomplete energy relaxation is explained by weak coupling between the states, especially between the corresponding states of absorption band I and II. The weak coupling is explained by the fact that these two states belong to different layers of the double-layer wall of the tubes [25].

Tubular J-aggregates as presented in Figure 1 were used to be decorated with metal nanoparticles. In a first approach, the palladium salt $\mathrm{Na}_{2} \mathrm{PdCl}_{4}$ was added to a solution of $\mathrm{C} 8 \mathrm{~S} 3$ aggregates. In Figure 2, the absorption spectra of a $5 \times 10^{-6} \mathrm{M}, \mathrm{C} 8 \mathrm{~S} 3$ solution in a $\mathrm{MeOH} /$ water mixture $(15 \mathrm{wt} \% \mathrm{MeOH})$ are presented for an increasing amount of $\mathrm{Na}_{2} \mathrm{PdCl}_{4}$. The spectra were recorded at titration steps of $1 \times 10^{-6} \mathrm{~mol} / \mathrm{l}$, starting with $1 \times 10^{-6} \mathrm{~mol} / \mathrm{l}$. The final concentration was $6 \times 10^{-6} \mathrm{~mol} / \mathrm{l}$ which is only a slight molar excess of $\left(\mathrm{PdCl}_{4}\right)^{2-}$ ions with respect to dye molecules. The addition of $\mathrm{Na}_{2} \mathrm{PdCl}_{4}$ causes not only a decrease of total absorbance, but also a qualitative change of the absorption spectrum. The most striking change is the disappearance of the absorption peaks II and IV at $590 \mathrm{~nm}$ and $560 \mathrm{~nm}$, respectively, while the band I at $600 \mathrm{~nm}$ is only slightly shifted and almost constant in intensity. At high concentrations of $\mathrm{Na}_{2} \mathrm{PdCl}_{4}$, the spectrum exhibits only two distinct bands. Absorption spectra of similar shape were found previously for tubular aggregates of another amphiphilic dye (a carboxyl substituted dye, named $\mathrm{C} 8 \mathrm{O} 3$ ) when additives like alcohols or surfactants were added $[28,29]$. As in the previous case, the spectral changes must be interpreted as the result of some 


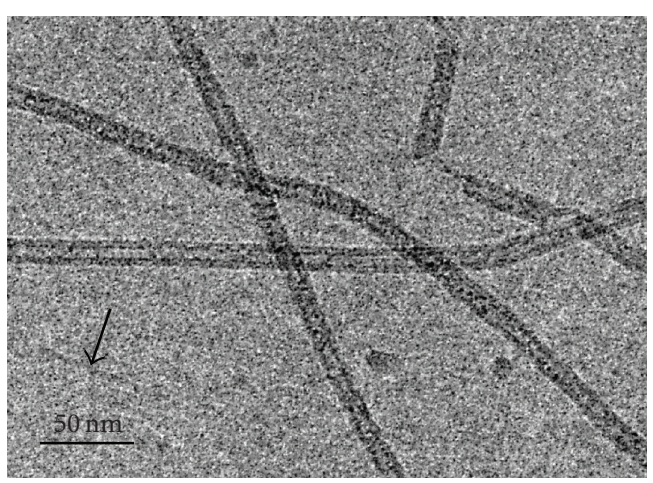

(a)

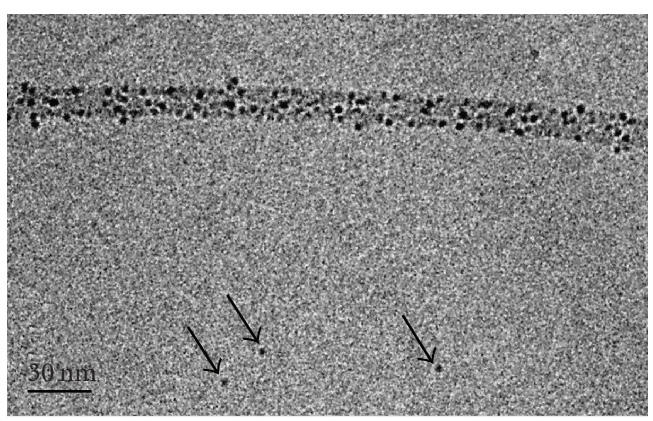

(b)

Figure 3: (a) Cryo-TEM image of C8S3 aggregates after addition of $\mathrm{Na}_{2} \mathrm{PdCl}_{4}$ without any redactor $\left([\mathrm{C} 8 \mathrm{~S} 3]=5 \times 10^{-4} \mathrm{~mol} \cdot \mathrm{l}^{-1}\right.$; $\left.\left[\mathrm{Na}_{2} \mathrm{PdCl}_{4}\right]=1.3 \times 10^{-6} \mathrm{~mol} \cdot \mathrm{l}^{-1}\right)$. (b) Same conditions as in (a), but after addition of reductor.

modifications of the arrangement of the molecules within the tubular wall, but with a maintenance of the tubular shape of the aggregate. According to the model of Didraga [25], one might conclude that the vanishing of band II is due to the destruction of the outer monolayer of the tubules wall due to the reduction of $\left(\mathrm{PdCl}_{4}\right)^{2-}$.

Inspection of the samples by cryo-TEM reveals that the aggregates are randomly covered by small palladium particles with an average diameter of $1.7 \pm 0.5 \mathrm{~nm}$. In Figure 3(a), a representative cryo-TEM micrograph is shown for a molar ratio of $\mathrm{Na}_{2} \mathrm{PdCl}_{4} / \mathrm{C} 8 \mathrm{~S} 3$ of $1: 3$. In this concentration regime, the absorption spectrum of the aggregate solution is almost unchanged (cf. Figure 2). The particles, identified by the black dots, are not very homogeneously distributed among the aggregates. There are even parts of the aggregates that are not at all covered with particles, for example, the horizontally oriented aggregate in the middle of Figure 3(a). Additionally, some of the aggregates are destroyed and the diameter along the aggregates becomes nonuniform. Due to destruction of the aggregates, pieces of the tubes can be identified within the image, as indicated by the arrow. It is also apparent from the image that the aggregates when decorated with Pd particles are softening. If an additional reducing agent is added to the solutions, larger Pd particles are found and the coverage of the aggregates with particles is increased significantly, as can be seen in the image of Figure 3(b). The size distribution of the particles is rather monodispers with a mean value of $2.7 \pm 0.2 \mathrm{~nm}$. Besides the increased coverage of the aggregates, also free particles in the solution are found as indicated by the arrows in Figure 3(b). But again, many of the aggregates are destroyed and the typical double-wall structure of the tubes is no longer visible.

There are four remarkable observations that can be drawn from these images. (1) The Pd particles are growing almost only on the surface of the aggregates. (2) The decoration of the aggregates with Pd softens and destroys the aggregate structure. This is in accordance with the spectroscopic changes described above. (3) Pd particles can be grown even without any secondary reductor. In this case, the aggregates are acting as reductor and are supposed to be oxidized. (4) The total number and the mean size of Pd particles is increased upon addition of a secondary reductor. Obviously, the reductor facilitates nucleation and growth of the particles.

Item (2) is of great importance because it gives evidence that the two electrons needed to reduce $\mathrm{Pd}(\mathrm{II})$ in the $\left(\mathrm{PdCl}_{4}\right)^{2-}$ complex to the zero valent state $\mathrm{Pd}^{0}$ are transferred from the dye aggregates to the Pd ions. Such an electron transfer is plausible since cyanine dye aggregates are widespread in use as photosensitizer in silver halide photography, where after photoexcitation an electron is transferred into the silver halide crystal $[30,31]$. Since all experiments were performed under room light conditions and comparable experiments in the dark are missing, so far we can only speculate that the reduction of $\mathrm{Pd}(\mathrm{II})$ is at least assisted by a photoexcited electron transfer (PET).

The addition of $\mathrm{Na}_{2} \mathrm{PdCl}_{4}$ to solutions of $\mathrm{C} 8 \mathrm{~S} 3$ aggregates causes significant fluorescence quenching. In Figure 4, the corresponding fluorescence spectra for the set of absorption spectra of Figure 2 are shown. The fluorescence intensity was corrected for reabsorption. The main reduction of fluorescence intensity (more than $85 \%$ of initial intensity) occurs at the first titration step, when $1 \times 10^{-6} \mathrm{~mol} / 1 \mathrm{Na}_{2} \mathrm{PdCl}_{4}$ is added to the $5 \times 10^{-6} \mathrm{M}$ dye solution. In this concentration regime of $\mathrm{Na}_{2} \mathrm{PdCl}_{4}$, no changes of the absorption spectrum is observed, as can be seen in Figure 2. For increasing quencher concentration, the shape of the fluorescence spectrum changes only slightly to that effect that the shoulder at $590 \mathrm{~nm}$ vanishes and the main peak shifts by approximately $2 \mathrm{~nm}$ towards the red. The disappearance of the shoulder is in accordance with the decrease of peak II in the absorption spectrum and the small shift of the main fluorescence peak is in parallel to the same shift of peak I. The small shoulder at $590 \mathrm{~nm}$ in the fluorescence spectra compared to the large peak II in the absorption spectrum indicates that most of the excitation energy is transferred into the lowest energy state of the aggregate, represented by the absorption/fluorescence band at $600 \mathrm{~nm}$. Since the emission properties of this peak do not change significantly and since the intensity of the shoulder at $590 \mathrm{~nm}$ is negligible, the emission behaves as it comes from a species which does not change its properties with quencher concentration. Therefore, we assume that the quenching of the fluorescence can be interpreted (at least in 


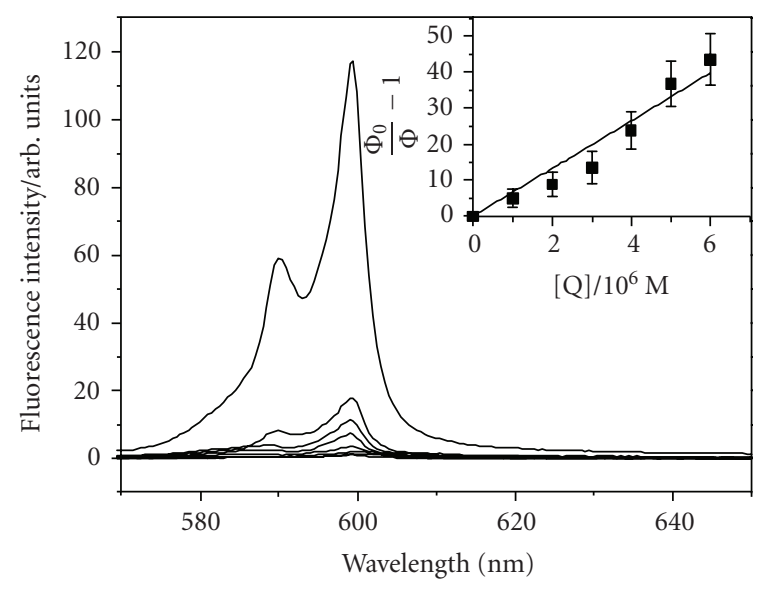

FIgURE 4: Fluorescence emission spectra of C8S3 aggregates for increasing concentrations of $\mathrm{Na}_{2} \mathrm{PdCl}_{4}$; corresponding to the absorption spectra of Figure 2. Excitation wavelength was $550 \mathrm{~nm}$. Insert: Stern-Vollmer plot of relative fluorescence intensity $\Phi / \Phi_{0}$ versus quencher concentration $[\mathrm{Q}]$, which is assumed to be the concentration of $\mathrm{Na}_{2} \mathrm{PdCl}_{4}$.

a first approximation) by a Stern-Volmer analysis as it is used in cases of bimolecular static or dynamic quenching [32].

In the insert of Figure 4, a Stern-Volmer plot of the relative fluorescence quantum efficiency is presented. As a raw estimate one can linearize the Stern-Volmer data and from the relationship

$$
\frac{\Phi_{0}}{\Phi}=1+K_{\mathrm{SV}} \cdot[\text { quencher }]
$$

a Stern-Volmer quenching constant $K_{\mathrm{SV}}$ of $6 \times 10^{6} \mathrm{M}^{-1}$ is obtained. This value is approximately 5 orders of magnitude higher than values reported in the case of conventional bimolecular static quenching and gives clear evidence for super quenching. Similar values are obtained by other authors for dye aggregates [18] or conjugated polymers [19], and values up to $10^{11} \mathrm{M}^{-1}$ were found for conjugated polymers where gold nanoparticles were used as quenchers [33].

So far it is an open question, if the fluorescence quenching is caused by the reduction reaction itself, that is, by an electron transfer to the $\mathrm{Pd}(\mathrm{II})$ ions, or if the metallic nanoparticles are acting as quenchers. In the latter case, the concentration of quenchers would be lower by roughly a factor of thousand which is approximately the mean number of $\mathrm{Pd}$ atoms per particle. The quenching constant has to be increased by the same number which results in very efficient superquenching. However, the simple Stern-Volmer analysis is questionable because of the inhomogeneous distribution of the Pd particles along the aggregates and the destruction of the aggregates by the Pd particles.

With this respect recent findings using $\mathrm{AgNO}_{3}$ instead of $\mathrm{Na}_{2} \mathrm{PdCl}_{4}$ are very prospective. Silver particles could be grown by the same mechanism, that is, reduction of $\mathrm{Ag}(\mathrm{I})$ ions into elementary silver. As in the case of palladium the addition of auxiliary reductor was assisting the formation of particles. In Figure 5, a representative cryo-TEM image of a

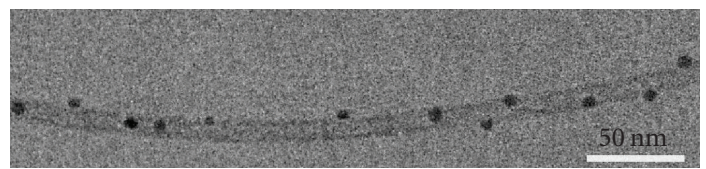

FIGURE 5: Cryo-TEM image of C8S3 aggregates after addition of $\mathrm{AgNO}_{3}$ and reducing agent. [C8S3] $=5 \times 10^{-4} \mathrm{~mol} \cdot \mathrm{l}^{-1}$ in water and $15 \mathrm{wt} \% \mathrm{MeOH} ;\left[\mathrm{AgNO}_{3}\right]=2.1 \times 10^{-5} \mathrm{~mol} \cdot \mathrm{l}^{-1}$.

solution of $\mathrm{C} 8 \mathrm{~S} 3$ aggregates after addition of $\mathrm{AgNO}_{3}$ and reductor is shown. Here, the tubular structure of the aggregates is well maintained and along the surface a few isolated silver nanoparticles are attached. In this case, the molar ratio Ag : dye was only $1: 23$, which favors the growth of isolated and well-separated particles. The size of the particles is very polydispers with a mean diameter in the range of 5-20 nm and the mean distance between particles is estimated to be in the region of 50 to $100 \mathrm{~nm}$. Fluorescence quenching was observed upon growth of the Ag nanoparticles and the absorption spectra are changing only slightly. Preliminary experiments show that the particles are formed only under illumination of the solutions under daylight. When they are kept completely in the dark, no formation of silver particles was observed by absorption spectroscopy which is taken as evidence that the reduction of silver is induced by light.

Further indication for a PET reaction may be drawn from the reduction and oxidation potentials of the cyanine dye. These potentials were measured for the monomer in acetonitrile against saturated calomel electrode (SCE) and reported to be $\mathrm{E}_{\mathrm{ox}}=0.6 \mathrm{~V}$ and $\mathrm{E}_{\mathrm{red}}=-1.61 \mathrm{~V}$ [34]. At least for the case of the silver ions, this oxidation potential of the dye would prohibit a spontaneous reduction of $\mathrm{Ag}^{+}$ions $\left(\mathrm{E}_{\text {red }}=0.56 \mathrm{~V}\right.$ against SCE $)$. However, for a detailed discussion, the exact potentials of the aggregates in aqueous solutions have to be known, the determination of which is a topic of our current research and beyond this publication.

\section{SUMMARY AND CONCLUSIONS}

The addition of metal salts such as $\mathrm{Na}_{2} \mathrm{PdCl}_{4}$ or $\mathrm{AgNO}_{3}$ to aqueous solutions of tubular J-aggregates of a tetrachlorobenzimidacarbocyanine dye leads to the formation of metal particles on the surface of the aggregates. The particles grown on the aggregate surface are of nanometer size and show a rather narrow size distribution. While palladium particles were growing in high density, silver particles were obtained in much less quantity but with larger size and broader size distribution, respectively. The particles are synthesized via a reduction reaction of the metal cations. Since the reduction occurs in presence of the dye aggregates, it is assumed that oxidation of the dyes is the elementary step of the particle synthesis. From preliminary experiments using samples that are prepared completely in the dark and those prepared at room light conditions, it is concluded that the reduction is due to a photo-induced electron transfer (PET). To our knowledge, this would be the first direct proof of a 
photo-induced electron transfer from a dispersion of mesoscopic J-aggregates in aqueous solution.

The particle growth leads to efficient quenching of the aggregate fluorescence. A simple Stern-Volmer analysis indicates super quenching. However, since it is not clear, if the quenching occurs from the electron transfer to the metal ions or by the metal particles, the Stern-Volmer picture is too simplistic. If the quenching results from particle only, then this analysis underestimates the quenching efficiency by orders of magnitude. In this case, the typical distance of energy migration could be obtained from the fluorescence quenching data in combination with a direct measurement of typical interparticle distances. However, controllable and homogeneous coverage of aggregates by metal particles would be a prerequisite which has not been achieved so far and is a topic of current studies.

\section{REFERENCES}

[1] G. Scheibe, "Über die veränderlichkeit des absorptionsspektrums einiger sensibilisierungsfarbstoffe und deren ursache," Angewandte Chemie, vol. 49, p. 563, 1936.

[2] E. E. Jelly, "Spectral absorption and fluorescence of dyes inthe molecular state," Nature, vol. 138, pp. 1009-1010, 1936.

[3] S. Dähne, "Der Mechanismus der photographischen Desensibilisierung," Zeitschift für Wissenschaftliche Photographie, photophysik und photochemie, vol. 59, pp. 113-173, 1965.

[4] A. S. Davydov, Theory of Molecular Excitons, Plenum Press, New York, NY, USA, 1971.

[5] V. Czikklely, H. D. Forsterling, and H. Kuhn, "Extended dipole model for aggregates of dye molecules," Chemical Physics Letters, vol. 6, no. 3, pp. 207-210, 1970.

[6] J. Knoester, "Nonlinear optical line shapes of disordered molecular aggregates: motional narrowing and the effect of intersite correlations," The Journal of Chemical Physics, vol. 99, no. 11, pp. 8466-8479, 1993.

[7] S. de Boer, K. J. Vink, and D. A. Wiersma, "Optical dynamics of condensed molecular aggregates: an accumulated photonecho and hole-burning study of the J-aggregate," Chemical Physics Letters, vol. 137, no. 2, pp. 99-106, 1987.

[8] F. C. Spano and S. Mukamel, "Superradiance in molecular aggregates," The Journal of Chemical Physics, vol. 91, no. 2, pp. 683-700, 1989.

[9] F. Meinardi, M. Cerminara, A. Sassella, R. Bonifacio, and R. Tubino, "Superradiance in molecular H aggregates," Physical Review Letters, vol. 91, no. 24, Article ID 247401, 4 pages, 2003.

[10] T. Kobayashi, Ed., J-Aggregates, World Scientific, Singapore, 1996.

[11] J. Moll, W. J. Harrison, D. V. Brumbaugh, and A. A. Muenter, "Exciton annihilation in $J$-aggregates probed by femtosecond fluorescence upconversion," Journal of Physical Chemistry A, vol. 104, no. 39, pp. 8847-8854, 2000.

[12] V. Sundström, T. Gillbro, R. A. Gadonas, and A. Piskarskas, "Annihilation of singlet excitons in $J$-aggregates of pseudoisocyanine (PIC) studied by pico- and subpicosecond spectroscopy," The Journal of Chemical Physics, vol. 89, no. 5, pp. 2754-2762, 1988.

[13] J. Moll, S. Dähne, J. R. Durrant, and D. A. Wiersma, "Optical dynamics of excitons in J-aggregates of a carbocyanine dye," The Journal of Chemical Physics, vol. 102, no. 16, pp. 63626370, 1995.
[14] I. G. Scheblykin, O. Y. Sliusarenko, L. S. Lepnev, A. G. Vitukhnovsky, and M. Van der Auweraer, "Strong nonmonotonous temperature dependence of exciton migration rate in $J$ aggregates at temperatures from 5 to $300 \mathrm{~K}$," Journal of Physical Chemistry B, vol. 104, no. 47, pp. 10949-10951, 2000.

[15] K. Ohta, M. Yang, and G. R. Fleming, "Ultrafast exciton dynamics of $J$-aggregates in room temperature solution studied by third-order nonlinear optical spectroscopy and numerical simulation based on exciton theory," The Journal of Chemical Physics, vol. 115, no. 16, pp. 7609-7621, 2001.

[16] G. Scheibe, A. Schöntag, and F. Katheder, "Fluoreszenz und Energiefortleitung bei reversibel polymerisierten Farbstoffen," Naturwissenschaften, vol. 27, no. 29, pp. 499-501, 1939.

[17] R. M. Jones, L. Lu, R. Helgeson, T. S. Bergstedt, D. W. McBranch, and D. G. Whitten, "Building highly sensitive dye assemblies for biosensing from molecular building blocks," Proceedings of the National Academy of Sciences of the United States of America, vol. 98, no. 26, pp. 14769-14772, 2001.

[18] L. Lu, R. Helgeson, R. M. Jones, D. McBranch, and D. Whitten, "Superquenching in cyanine pendant poly(L-lysine) dyes: dependence on molecular weight, solvent, and aggregation," Journal of the American Chemical Society, vol. 124, no. 3, pp. 483-488, 2002.

[19] C. Tan, E. Atas, J. G. Müller, M. R. Pinto, V. D. Kleiman, and K. S. Schanze, "Amplified quenching of a conjugated polyelectrolyte by cyanine dyes," Journal of the American Chemical Society, vol. 126, no. 42, pp. 13685-13694, 2004.

[20] A. Pawlik, S. Kirstein, U. De Rossi, and S. Dähne, "Structural conditions for spontaneous generation of optical activity in $J$ aggregates," Journal of Physical Chemistry B, vol. 101, no. 29, pp. 5646-5651, 1997.

[21] S. Kirstein, H. von Berlepsch, C. Böttcher, et al., "Chiral Jaggregates formed by achiral cyanine dyes," ChemPhysChem, vol. 1, no. 3, pp. 146-150, 2000.

[22] H. von Berlepsch, C. Böttcher, A. Ouart, et al., "Surfactantinduced changes of morphology of $J$-aggregates: superhelixto-tubule transformation," Langmuir, vol. 16, no. 14, pp. 5908-5916, 2000.

[23] H. von Berlepsch, M. Regenbrecht, S. Dähne, S. Kirstein, and C. Böttcher, "Surfactant-induced separation of stacked Jaggregates. Cryo-transmission electron microscopy studies reveal bilayer ribbons," Langmuir, vol. 18, no. 7, pp. 2901-2907, 2002.

[24] A. Pawlik, A. Ouart, S. Kirstein, H.-W. Abraham, and S. Dähne, "Synthesis and UV/Vis spectra of $J$-aggregating $5,5^{\prime}, 6,6^{\prime}$-tetrachlorobenzimidacarbocyanine dyes for artificial light-harvesting systems and for asymmetrical generation of supramolecular helices," European Journal of Organic Chemistry, no. 16, pp. 3065-3080, 2003.

[25] C. Didraga, A. Pugžlys, P. R. Hania, H. von Berlepsch, K. Duppen, and J. Knoester, "Structure, spectroscopy, and microscopic model of tubular carbocyanine dye aggregates," Journal of Physical Chemistry B, vol. 108, no. 39, pp. 14976-14985, 2004.

[26] G. O. Mallory and J. B. Hajdu, Electroless Plating, American Electroplaters and Surface Finishers Society, Orlando, Fla, USA, 1989.

[27] G. M. Chow, M. Pazurandeh, S. Baral, and J. R. Campbell, "TEM and HRTEM characterization of metallized nanotubules derived from bacteria," Nanostructured Materials, vol. 2, no. 5, pp. 495-503, 1993. 
[28] H. von Berlepsch, S. Kirstein, R. Hania, A. Pugžlys, and C. Böttcher, "Modification of the nanoscale structure of the Jaggregate of a sulfonate substituted amphiphilic carbocyanine dye through incorporation of surface-active additives," Journal of Physical Chemistry B, in press.

[29] H. von Berlepsch, S. Kirstein, and C. Böttcher, "Effect of alcohols on J-aggregation of a carbocyanine dye," Langmuir, vol. 18, no. 20, pp. 7699-7705, 2002.

[30] T. Tani and Y. Sano, "Electro-spin resonance study of positive holes in $J$-aggregates of a cyanine dye on AgBr microcrystals: effect of aggregate size," Journal of Applied Physics, vol. 69, no. 8, pp. 4391-4397, 1991.

[31] B. Trösken, F. Willig, K. Schwarzburg, A. Ehert, and M. Spitler, "Electron transfer quenching of excited $J$-aggregate dyes on $\mathrm{AgBr}$ microcrystals between 300 and $5 \mathrm{~K}$," Journal of Physical Chemistry, vol. 99, no. 14, pp. 5152-5160, 1995.

[32] J. R. Lakowicz, Principles of Fluorescence Spectroscopy, Kluwer Academic, New York, NY, USA, 1999.

[33] C. Fan, S. Wang, J. W. Hong, G. C. Bazan, K. W. Plaxco, and A. J. Heeger, "Beyond superquenching: hyper-efficient energy transfer from conjugated polymers to gold nanoparticles," Proceedings of the National Academy of Sciences of the United States of America, vol. 100, no. 11, pp. 6297-6301, 2003.

[34] K. Hosoi, A. Hirano, and T. Tani, "Dynamics of photocreated positive holes in silver bromide microcrystals with adsorbed cyanine dyes," Journal of Applied Physics, vol. 90, no. 12, pp. 6197-6204, 2001. 


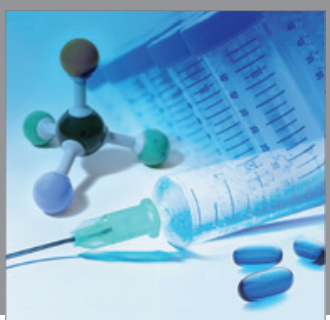

International Journal of

Medicinal Chemistry

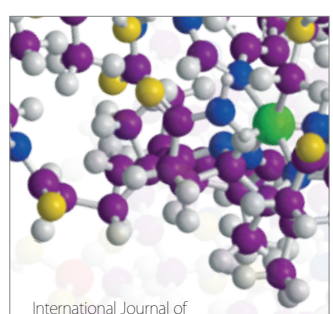

Carbohydrate Chemistry

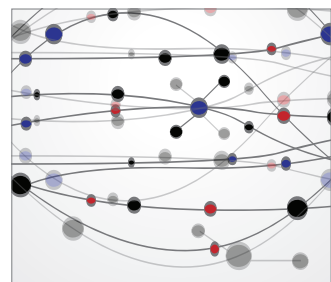

The Scientific World Journal
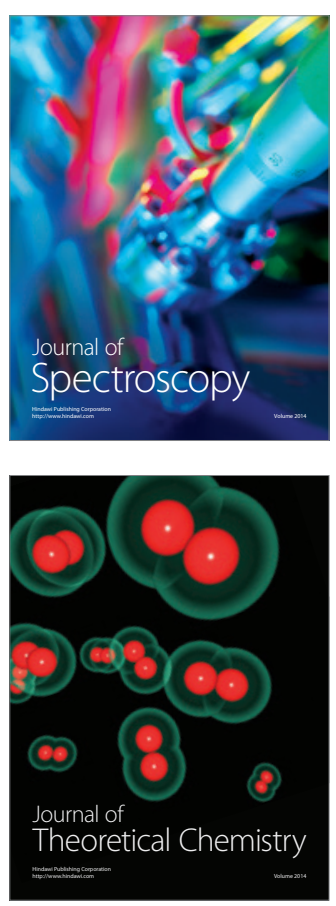
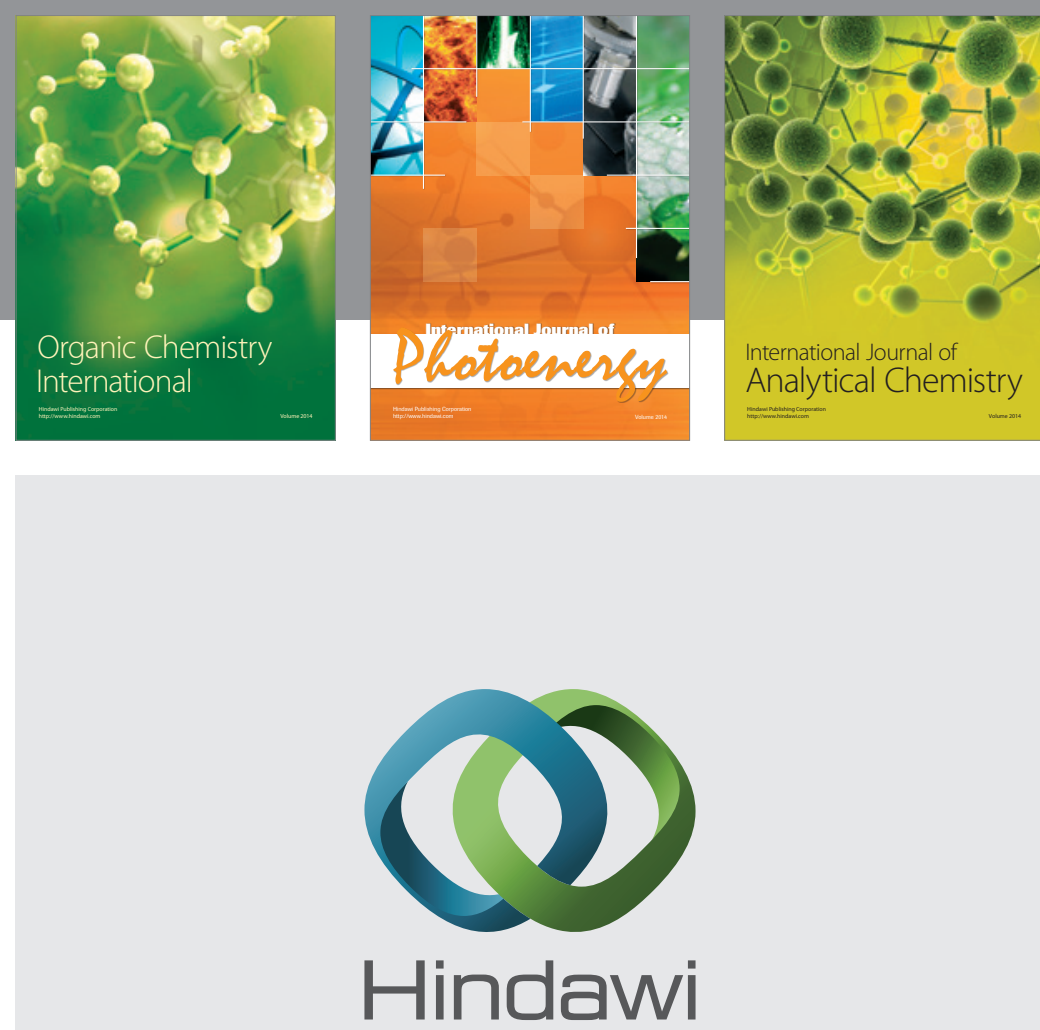

Submit your manuscripts at

http://www.hindawi.com
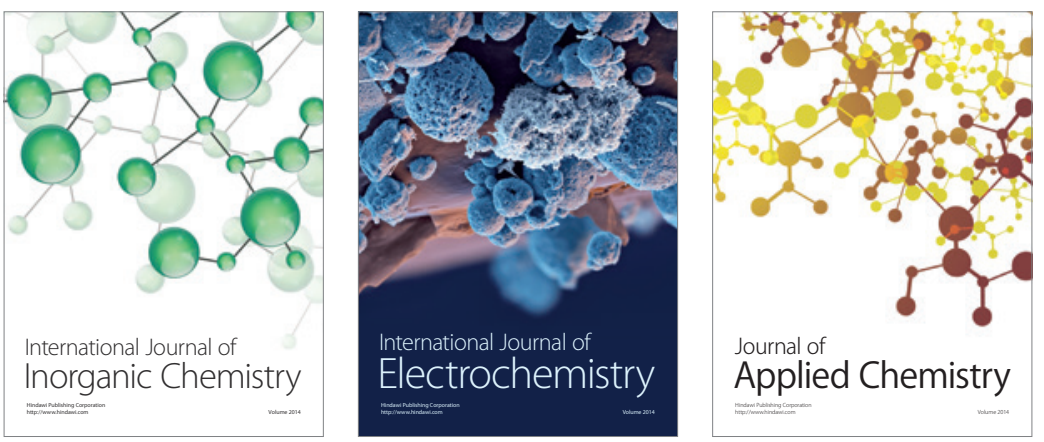

Journal of

Applied Chemistry
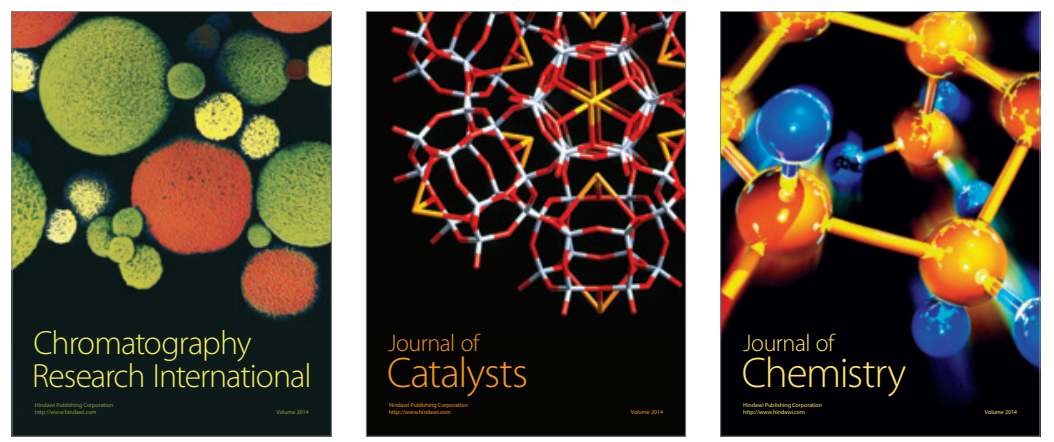
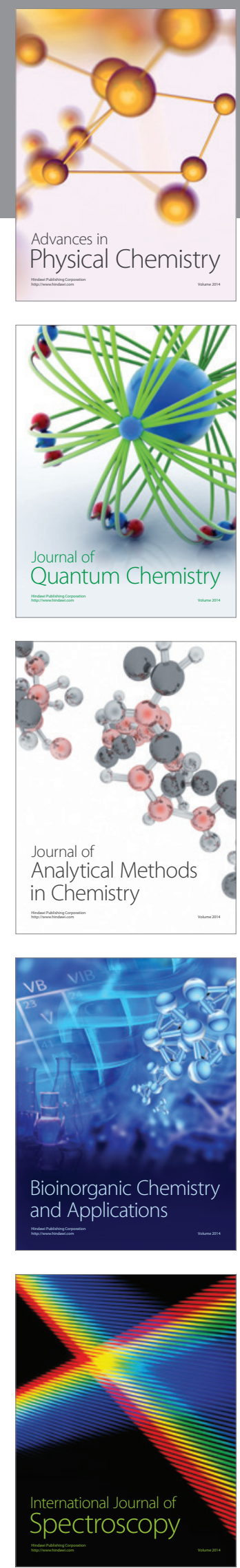